\title{
ESBL DETECTION: PREVALENCE \& COMPARISON WITH NEW CRITERIA
}

Dr. Ashwini Manhas, Dr. Prerna Aggarwal, Dr. Manju Bala, Dr. Satish Gupte.

1. Assistant Professor, Department of Microbiology, Giansagar Medical College, Patiala (Punjab).

2. Associate Professor, Department of Microbiology, Giansagar Medical College, Patiala (Punjab).

3. Assistant Professor, Department of Microbiology, Giansagar Medical College, Patiala (Punjab).

4. Professor, Department of Microbiology, Giansagar Medical College, Patiala (Punjab).

\section{CORRESPONDING AUTHOR}

Dr. Ashwini Manhas

Giansagar Medical College,Ramnagar,

Rajpura,Dist. Patiala (Punjab) Pin code-140601

E-mail: drashwinimanhas@yahoo.com,

Ph: 09888473208.

ABSTRACT: AIM: To study the prevalence of ESBL producing organisms in our area both by phenotypic method and latest CLSI breakpoints. Effect of change in break points on predicting ESBL was also studied. MATERIAL \& METHODS: Successive clinical isolates were tested by phenotypic assay and zone diameters compared by Kirby Bauer's disc diffusion method.

RESULTS: Total 231 clinical isolates were studied of which $81.8 \%$ were ESBL producers. Cefotaxime had high sensitivity (97.91\%), specificity (97.43\%) and positive predictive value (99.47\%). Cefpodoxime had strikingly $100 \%$ specificity and positive predictive value but a very poor negative predictive value. Aztreonam had a very high sensitivity (99.4\%) and negative predictive value (97.61\%) CONCLUSION: Cefotaxime was found to be the most appropriate antibiotic when used as single agent.

KEYWORDS: ESBL, Double Disc Synergy Test, Drug Resistance

INTRODUCTION: Since their first description more than twenty years ago, pathogens producing extended-spectrum $\beta$ - lactamases (ESBLs) have become an increasing cause of clinical concern for several reasons ${ }^{1-3}$. First, systemic infections due to ESBL-producing Enterobacteriaceae are associated with adverse clinical outcomes. Second, initially restricted to certain, these enzymes have spread globally and their prevalence varies geographically. Third, primarily characterized in limited bacteria such as Escherichia coli and Klebsiella spp., ESBLs have been spreading and reaching other genera, principally Enterobacter and Proteus spp. Finally, besides the growing species diversity, ESBL phenotypes have become more complex due to the production of multiple enzymes including inhibitor-resistant ESBL variants, plasmidborne AmpC, production of ESBLs in AmpC - producing bacteria, production of ESBLs in KPCproducing bacteria, enzyme hyperproduction and porin loss ${ }^{1-4}$.

A strong relationship exists between third-generation cephalosporin use and acquisition of an ESBL producing strain ${ }^{5}$. Other antibiotic classes associated with infections due to ESBLproducing organisms include quinolones, trimethoprim-sulphamethoxazole, aminoglycosides and metronidazole. ${ }^{6}$ In some centers from India as many as 86 percent of Klebsiellae have been found to be ESBL producers ${ }^{7}$.

Despite Clinical and Laboratory Standard Institute's (CLSI) recommendations 6,8 that clinical microbiology laboratories perform specialized tests for detection of ESBLs, many of them make no effort to detect ESBL production or are ineffective at doing so. In India there is no regulation regarding performance and reporting of ESBL isolates from various hospitals and 
clinical laboratories. Many laboratories lack sufficient expertise to identify and report ESBLs. There is a need for simple yet effective methodologies which can be routinely used for monitoring the prevalence of ESBL producing organisms.

The most common method of testing for extended spectrum $\beta$-lactamases (ESBLs) is screening for reduced susceptibility to cefpodoxime/ cefotaxime/ ceftriaxone/ ceftazidime/ aztreonam followed by phenotypic confirmatory testing by demonstrating a synergistic effect between an indicator cephalosporin and a $\beta$-lactamase inhibitor i.e., clavulanic acid 9,10. The sensitivity of screening for ESBLs can vary depending on the type of antimicrobial agent tested.11, 12 According to Clinical Laboratory Standards Institute (CLSI) M100-S13 guidelines, ${ }^{10}$ use of more than one of the five indicator cephalosporins suggested for screening improves the sensitivity of ESBL detection. Some studies have reported that if it should be necessary to rely on a single screening antibiotic, cefpodoxime or ceftazidime is the recommended antibiotic for ESBL detection in Klebsiella spp.11,12 Phenotypic methods are easier to perform as compared to genotypic and at the same time they are indicative of gene expression. If criteria for ESBL detection could be incorporated in routine susceptibility testing the detection and interpretation would become simpler.

So a study was planned to estimate the prevalence of ESBL producing organisms in our area both by phenotypic method and latest CLSI breakpoints. The isolates studied belonged to family Enterobacteriacae.

MATERIALS \& METHODS: The study was conducted in Giansagar Medical College and Hospital which is a 600 bedded tertiary care hospital. A prospective study on successive clinical isolates of family enterobacteriacae was undertaken. All isolates were screened for ESBL production by Kirby- Bauer's disc diffusion method demonstrating reduced susceptibility to cefpodoxime (30 $\mu \mathrm{g})$, cefotaxime $(30 \mu \mathrm{g})$, ceftazidime $(30 \mu \mathrm{g})$, and aztreonam $(30 \mu \mathrm{g})$. Cut-off zone sizes as an indicator of potential ESBL producer for K. pneumoniae, K. oxytoca \& E. coli were $\leq 27 \mathrm{~mm}$ for cefotaxime, $\leq 22 \mathrm{~mm}$ for ceftazidime, $\leq 27 \mathrm{~mm}$ for aztreonam and $\leq 17 \mathrm{~mm}$ for cefpodoxime. For P. mirabilis the zone size cut offs were cefotaxime $\leq 27 \mathrm{~mm}$, ceftazidime $\leq 22 \mathrm{~mm}$, aztreonam $\leq$ $27 \mathrm{~mm}$ and cefpodoxime $\leq 22 \mathrm{~mm}$. Escherichia coli ATCC 25922 \& Klebsiella pneumoniae ATCC 700603 (ESBL positive) were used as quality control for ESBL detection.

Phenotypic confirmatory test was performed for confirmation. The combined disk method was used to confirm the presence of ESBL on all the isolates of enterobacteriacae family by placing a disk of ceftazidime $(30 \mu \mathrm{g})$ and ceftazidime $(30 \mu \mathrm{g}) /$ clavulanic acid $(10 \mu \mathrm{g})$ besides cefotaxime $(30 \mu \mathrm{g})$ and cefotaxime $(30 \mu \mathrm{g}) /$ clavulanic acid $(10 \mu \mathrm{g})$ on a Mueller-Hinton agar plate as recommended by CLSI. A $\geq 5 \mathrm{~mm}$ increase in zone diameter for either antimicrobial agent tested in combination with clavulanic acid vs its zone when tested alone was considered ESBL producer. CLSI has reviewed the interpretation criteria as shown in Table $2 .{ }^{18}$ If new interpretation criteria are being used then the screening test need not be performed. Penicillins, cephalosporins and aztreonam should be reported as found on susceptibility testing without altering susceptibility status as recommended earlier.

RESULTS: Altogether 231 clinical isolates were studied. The most prevalent isolate was E. coli (78.3\%) followed by Klebsiella spp.(12.1\%), Proteus mirabilis (4.3\%), Citrobacter spp. (2.1\%) \& others (Table 1). Overall ESBL producers were $81.8 \%$ and ESBL non producers were $18.2 \%$. Outpatient clinics contributed to 65 isolates of total 231 isolates. Amongst them the prevalence of ESBLs was 75.38\% (49/65). From inpatients 166 isolates were obtained amongst which the prevalence of ESBLs was 84.34\% (140/166) (Table 3).

Journal of Evolution of Medical and Dental Sciences/Volume1/ Issue3/July-Sept 2012 Page 210 
TABLE 1: Species distribution

\begin{tabular}{lll}
\hline Species & No. of isolates & ESBL producers \\
\hline Escherichia coli & $181(78.3 \%)$ & $150(82.8 \%)$ \\
Klebsiella species & $28(12.1 \%)$ & $22(78.5 \%)$ \\
Proteus mirabilis & $10(4.3 \%)$ & $8(80 \%)$ \\
Citrobacter species & $5(2.1 \%)$ & $5(100 \%)$ \\
Serratia species & $4(1.7 \%)$ & $3(75 \%)$ \\
$\begin{array}{l}\text { Enterobacter } \\
\text { species }\end{array}$ & $1(0.4 \%)$ & $1(100 \%)$ \\
$\begin{array}{l}\text { Morganella } \\
\text { morganii }\end{array}$ & $1(0.4 \%)$ & $1(100 \%)$ \\
\begin{tabular}{l} 
Salmonella typhi \\
\hline
\end{tabular} & $1(0.4 \%)$ & $0(0 \%)$ \\
\hline
\end{tabular}

TABLE 2: Revised CLSI zone diameter (in $\mathrm{mm}$ )

\begin{tabular}{llll}
\hline & Sensitive & Intermediate & Resistance \\
\hline Cefotaxime & $\geq 26$ & $23-25$ & $\leq 22$ \\
Ceftazidime & $\geq 21$ & $18-20$ & $\leq 17$ \\
Aztreonam & $\geq 21$ & $18-20$ & $\leq 17$ \\
Cefpodoxime & $\geq 21$ & $18-20$ & $\leq 17$ \\
\hline
\end{tabular}

TABLE 3: In patient \& outpatient distribution

\begin{tabular}{llll}
\hline & Isolates & ESBL producers & $\begin{array}{l}\text { Non ESBL } \\
\text { producers }\end{array}$ \\
\hline Out patient & 65 & $49(75.38 \%)$ & $16(24.61 \%)$ \\
In patient & 166 & $140(84.34 \%)$ & $26(15.66 \%)$ \\
Total & 231 & $189(81.80 \%)$ & $42(18.18 \%)$ \\
\hline
\end{tabular}

TABLE 4: Comparison of old and new CLSI criteria for ESBL detection

\begin{tabular}{lllllllll}
\hline & \multicolumn{2}{l}{ Sensitivity } & \multicolumn{2}{l}{ Specificity } & \multicolumn{2}{l}{$\begin{array}{l}\text { Positive } \\
\text { predictive value }\end{array}$} & $\begin{array}{l}\text { Negative } \\
\text { predictive value }\end{array}$ \\
\hline & $\begin{array}{l}\text { Old } \\
\text { criteria }\end{array}$ & $\begin{array}{l}\text { New } \\
\text { criteria }\end{array}$ & $\begin{array}{l}\text { Old } \\
\text { criteria }\end{array}$ & $\begin{array}{l}\text { New } \\
\text { criteria }\end{array}$ & $\begin{array}{l}\text { Old } \\
\text { criteria }\end{array}$ & $\begin{array}{l}\text { New } \\
\text { criteria }\end{array}$ & $\begin{array}{l}\text { Old } \\
\text { criteria }\end{array}$ & $\begin{array}{l}\text { New } \\
\text { criteria }\end{array}$ \\
Cefotaxime & $97.91 \%$ & $97.91 \%$ & $97.43 \%$ & $97.43 \%$ & $99.47 \%$ & $99.47 \%$ & $90.47 \%$ & $90.47 \%$ \\
Ceftazidime & $91.45 \%$ & $95.72 \%$ & $78.12 \%$ & $77.27 \%$ & $96.29 \%$ & $94.7 \%$ & $59.52 \%$ & $80.95 \%$ \\
Aztreonam & $89.9 \%$ & $99.4 \%$ & $91.3 \%$ & $66.12 \%$ & $98.94 \%$ & $88.88 \%$ & $50 \%$ & $97.61 \%$ \\
Cefpodoxime & $96.89 \%$ & $82.17 \%$ & $94.73 \%$ & $100 \%$ & $99.94 \%$ & $100 \%$ & $85.71 \%$ & $2.38 \%$ \\
\hline
\end{tabular}

The species distribution of ESBLs amongst the various isolates was as shown in Table 1. Using phenotypic methods the highest number of ESBLs was contributed by E. coli followed by Klebsiella spp. and Proteus mirabilis. Citrobacter spp. though had only 5 isolates but alarmingly all of them were ESBL producers. The drugs that were tested for ESBL production were cefotaxime, aztreonam, ceftazidime \& cefpodoxime. We also compared the zone diameters 
according to old and current CLSI criteria to see if a single drug was better predictor of ESBL activity when used in the absence of ESBL phenotypic assay.

The effect of change in CLSI criteria on predicting ESBL production is shown in Table 4. For cefotaxime the sensitivity, specificity, positive predictive value and negative predictive value was $97.91 \%, 97.43 \%, 99.47 \%$ \& $90.47 \%$ respectively. Upon testing with ceftazidime the sensitivity was $95.72 \%$ and positive predictive value was $94.7 \%$. A fall in specificity $(77.27 \%)$ and negative predictive value (80.95\%) was observed. With ceftazidime 182 isolates were confirmed ESBL producers, 25 were ESBL non producers and 24 were doubtful. Out of these isolates 17 were later found to be non ESBL producers and 7 were ESBL producers. With aztreonam 187 isolates were confirmed ESBL producers, 21 were non ESBL producers and 23 were doubtful. Of these 23, 21 isolates were found to be non ESBL producers but 2 were ESBL producers. Aztreonam showed improved sensitivity $(99.4 \%)$ and negative predictive value (97.61\%) but reduced specificity $(66.12 \%)$ and positive predictive value $(88.88 \%)$. For cefpodoxime the current criteria resulted in $100 \%$ specificity and positive predictive value but a poorer sensitivity $(82.17 \%)$ and negative predictive value $(2.38 \%)$.

DISCUSSION: ESBL producing bacteria have become quite prevalent in Indian hospitals and community. Different studies from India have quoted variable ESBL detection rates. According to multicentric SMART study conducted in Asia Pacific region during the year 2007 ESBL rates in India for E. coli, Klebsiella pneumoniae, and Klebsiella oxytoca were 79.0\%, 69.4\%, and $100 \%$, respectively ${ }^{13}$. Another study found ESBL producing E. coli increase from 40 per cent in 2002 to 61 per cent in 2009 , with a significant $(\mathrm{P}<0.05)$ rise in resistance to cefotaxime $(75$ to $97 \%$ ). This study also found the resistance directly related to high antibiotic usage. ${ }^{14} \mathrm{~A}$ study from central India reports the incidence of $69 \%$ and $41 \%$ ESBL producing isolates of E. coli and Klebsiella pneumoniae respectively. ${ }^{15}$ Another study from Vellore in South India found $50.29 \%$ of inpatients and $45.86 \%$ of outpatients harboring ESBL producers. ${ }^{16}$ A study from Northwestern India found ESBL production as $23.83 \%$ in E. coli strains and $8.69 \%$ in Klebsiella strains ${ }^{17}$. We observed that $81.8 \%$ of clinical isolates were ESBL producers. From outpatient clinics the reporting of ESBL producers was $75.38 \%$ whereas from inpatient clinics it was $84.34 \%$.

Though ESBLs are prevalent in our hospitals and community they are not specifically reported by Microbiology labs. For certain reasons there is also a wide variability in prevalence reporting of ESBLs. The possible factors could be different geographical locations, variable proficiency levels of microbiology trained technical staff, different antibiotic cut offs being used, different guidelines being followed (as they differ between different infectious diseases unions throughout the world) and different techniques being used for ESBL detection.

In developing countries like India it is desirable to incorporate both ESBL detection and antimicrobial susceptibility testing. Different drugs have been used to detect ESBLs but no single antibiotic has been perfect at ruling -in or -out an ESBL producer. CLSI has recently changed the breakpoints of for aztreonam, cefotaxime and ceftazidime for routine testing.

Criteria for cefotaxime to be used for ESBL detection have remained unchanged. With high sensitivity, specificity and positive predictive value it remains the best drug for ESBL detection. Ceftazidime has remained almost equally sensitive and specific as old criteria with improvement in negative predictive value from $59.52 \%$ to $80.95 \%$ with almost sustained sensitivity, specificity and positive predictive value. Aztreonam had shown improved sensitivity 
from $89.9 \%$ to $99.4 \%$ but reduction in specificity from $91.3 \%$ to $66.12 \%$. Its positive predictive value drops from $98.94 \%$ to $88.88 \%$ with significant increase in negative predictive value to $97.61 \%$. Therefore, to improve the interpretation aztreonam remains additional preferred agent besides cefotaxime. Cefpodoxime has shown reduced sensitivity from $96.89 \%$ to $82.17 \%$ with sustained improvement in specificity and positive predictive value to $100 \%$. The most striking observation was a very poor negative predictive value. This will result in need to perform additional confirmatory tests if used as single agent.

Overall we found that cefotaxime was the single most appropriate antibiotic for predicting ESBL production. Cefpodoxime can be considered a good agent due to high specificity and positive predictive value. However, it has a very poor negative predictive value. Therefore, no single agent can be recommended for predicting ESBL production.

CLSI has so far not given any specific criteria with the use of above mentioned four antibiotics for detecting ESBLs while performing routine susceptibility testing. At the same time it recommends that with the current criteria antibiotics should be reported as observed on susceptibility testing. Earlier it recommended the reporting of all drugs as resistant when it was observed as resistant to any one drug of the four. These guidelines are based on PK/PD analysis.

More clinical studies are needed to support the current recommendation which is based on PK/PD measurements of the above mentioned drugs. Therapeutic failure or success of antibiotic therapy when one drug is sensitive and other resistant in vitro needs to be studied in parallel with clinical outcome of cases.

To conclude, cefotaxime was found to be the most appropriate drug when used as single agent with both old as well as current criteria. Adding Aztreonam to the interpretation improves the detection of ESBL. The current criteria definitely reduce the need for ESBL screening test due to increased sensitivity and negative predictive value with at least three out of four antibiotics. However, the need for using more than one drug for predicting ESBL activity continues to be there as before.

ACKNOWLEDGEMENT: "We are thankful to the Management \& Principal, Giansagar Medical College, Patiala for approving and facilitating the study."

\section{REFERENCE:}

1. Kim YK, Pai H, Lee HJ, Park SE, Choi EH, Kim J, Kim JH, Kim EC. Bloodstream infections by extended-spectrum beta-lactamase-producing Escherichia coli and Klebsiella pneumoniae in children: epidemiology and clinical outcome. Antimicrob Agents Chemother 2002;46: 1481- 1491.

2. Schwaber MJ, Navon-Venezia JS, Kaye KS, Ben-Ami R, Schwartz D, Carmeli Y. Clinical and economic impact of bacteremia with extended-spectrum-\{beta\}-lactamase-producing Enterobacteriaceae. Antimicrob Agents Chemother 2006; 50: 1257-1262.

3. Perez F, Endimiani A, Hujer KM, Bonomo RA. The continuing challenge of ESBLs. Curr Opin Pharmacol 2007; 7: 459-469.

4. Tsakris A, Poulou A, Themeli-Digalaki K, Voulgari E, Pittaras T, Sofianou D, Pournaras S, Petropoulou D. Use of boronic acid disk tests to detect extended spectrum beta- 
lactamases in clinical isolates of KPC carbapenemase-possessing Enterobacteriaceae. J Clin Microbiol 2009; 47: 3420- 3426

5. Lautenbach E, Patel JB, Bilker WB, Edelstein PH, Fishman NO. Extended-spectrum betalactamase-producing Escherichia coli and Klebsiella pneumoniae: risk factors for infection and impact of resistance on outcomes. Clin Infect Dis 2001; 32: 1162-71.

6. Paterson DA, Bonomo RA. Extended-spectrum b-lactamases: a clinical update. Clin Microbiol Rev 2005; 18: 657-86.

7. Jain A, Mondal R. Prevalence \& antimicrobial resistance pattern of extended spectrum beta-lactamase producing Klebsiella spp. isolated from cases of neonatal septicemia. Indian J Med Res 2007; 125: 699-700.

8. Clinical and Laboratory Standards Institute. Performance standards for antimicrobial susceptibility testing. $13^{\text {th }}$ Informational Supplement. Wayne, Pennsylvania. CLSI Document M100-S13: 2003.

9. Sturenburg E, Mack D. Extended spectrum beta-lactamases: implications for the clinical microbiology laboratory, therapy, and infection control. J infect 2003; 47 : 273 -95.

10. Clinical and Laboratory Standards Institute. Performance standards for antimicrobial susceptibility testing; 16 $6^{\text {th }}$ Informational Supplement; Wayne, Pennsylvania. CLSI Document M100-S16; 2006.

11. MacKenzie FM, Miller CA, Gould IM. Comparison of screening methods for TEM- and SHV-derived extended spectrum beta-lactamase detection. Clin Microbiol Infect 2002; 8: 715-24.

12. Ho PL, Tsang DN, Ho M, Yuen KY. Comparison of screening methods for detection of extended spectrum beta-lactamases and their prevalence among Escherichia coli and Klebsiella species in Hong Kong. APIMS 2000; 108: 237-40.

13. Hawser SP, Bouchillon SK, Hoban DJ, Badal RE, Hsueh PR, Paterson DL, et al. Emergence of high levels of extended-spectrum-ß-lactamase-producing gram-negative bacilli in the Asia-Pacific region: data from the Study for Monitoring Antimicrobial Resistance Trends (SMART) program, 2007. Antimicrob Agents Chemother. 2009; 53: 3280-4

14. Datta S, Wattal C, Goel N, Oberoi JO, Raveendran R and Prasad KJ. A ten year analysis of multi-drug resistant blood stream infections caused by Escherichia coli \& Klebsiella pneumoniae in a tertiary care hospital. Indian J Med Res. 2012 June; 135(6): 907-912

15. Pathak A, Marothi Y, Kekre V, Mahadik K, Macaden R, Lundborg CS. High prevalence of extended-spectrum $\beta$-lactamase-producing pathogens: results of a surveillance study in two hospitals in Ujjain, India. Infect Drug Resist. 2012;5:65-73

16. Sankar S, Narayanan H, Kuppanan S, Nandagopal B. Frequency of extended-spectrum $\beta$ lactamase (ESBL)-producing Gram-negative bacilli in a 200-bed multi-specialty hospital in Vellore district, Tamil Nadu, India. Infection. 2012 Aug;40(4):425-9

17. Sood S, Gupta R. Antibiotic resistance pattern of community acquired uropathogens at a tertiary care hospital in jaipur, rajasthan. Indian J Community Med. 2012 Jan;37(1):3944

18. Clinical and Laboratory Standards Institute. Performance standards for antimicrobial susceptibility testing; 21 $1^{\text {st }}$ Informational Supplement; Wayne, Pennsylvania. CLSI Document M100-S21;

2011. 